\title{
A Atividade Física no Presente Pode Ser a Receita para Evitar os Males da Obesidade e Hipertensão no Futuro
}

\author{
Physical Activity in the Present Can Be the Recipe to Avoid the IIls of Obesity and Hypertension in the Future
}

\author{
Roberto Jorge da Silva Franco ${ }^{(1)}$ \\ Universidade Estadual Paulista Júlio de Mesquita Filho Câmpus de Botucatu Faculdade de Medicina, Botucatu, SP - Brasil \\ Minieditorial referente ao artigo: Medidas Hipertensivas em Escolares: Risco da Obesidade Central e Efeito Protetor da Atividade Física \\ Moderada-Vigorosa
}

A hipertensão arterial é uma das causas mais importantes de morte prematura no mundo. Quase um bilhão de pessoas são afetadas e estima-se que até 2025, 1,5 bilhão de adultos terão HA. ${ }^{1}$ Felizmente, a HA é um dos principais fatores de risco modificáveis para doenças cardiovasculares com raízes na infância, tornando evitáveis tanto o desenvolvimento da doença quanto o óbito.

Atualmente, a prevalência de HA em crianças e adolescentes tornou-se um importante problema de saúde pública. ${ }^{2}$ Um exemplo de como esse cenário se agravou é a comparação de um estudo feito pelo nosso grupo ${ }^{3}$ com duas coortes sucessivas (1975-1976) de estudantes de 16 a 25 anos de idade da cidade de Botucatu, estado de São Paulo, em que encontraram uma prevalência de $\mathrm{HA}$ de $5,0 \%$ e $6,2 \%$, respectivamente, além de outro estudo mais recente. Com quase 40 anos de atraso, o estudo brasileiro sobre riscos cardiovasculares em adolescentes (ERICA), ${ }^{4}$ um estudo escolar nacional que contou com 73.999 adolescentes com idades entre 12 e 17 anos, encontrou uma prevalência de HA de 9,6\%. Os adolescentes obesos apresentaram maior prevalência de hipertensão $28,4 \%$ - que os adolescentes com excesso de peso, com $15,4 \%$, e os eutróficos, com 6,3\%. A fração de HA atribuível à obesidade foi de 17,8\%.

Muitas razões podem explicar a crescente prevalência de HA em jovens. A obesidade é a principal, seguida pelo consumo de sal e açúcar, ambientes estressantes, baixo nível de atividade física e estilo de vida sedentário. No Brasil, um estudo transversal sobre consumo de alimentos ${ }^{5}$ em amostra representativa da população com 10 anos ou mais de idade observou que o maior consumo de alimentos ultraprocessados estava associado a maior teor de gorduras em geral, gordura saturada, gordura trans e açúcares livres.

As evidências da proteção contra o desenvolvimento de HA e doenças cardiovasculares e mortalidade por todas

\section{Palavras-chave}

Hipertensão; Fatores de Risco; Doenças Cardiovasculares/ mortalidade; Adolescentes; Adulto; Obesidade; Atividade Física; Sedentarismo.

Correspondência: Roberto Jorge da Silva Franco •

Universidade Estadual Paulista Júlio de Mesquita Filho Câmpus de Botucatu Faculdade de Medicina - Av. Professor Montenegro, s/n. CEP 18618-687,

Botucatu, SP - Brasil

E-mail: roberto.js.franco@unesp.br

DOI: https://doi.org/10.36660/abc.20200483 as causas por parte da atividade física regular tornaram-se incontestáveis. ${ }^{6} \mathrm{~A}$ maioria das pessoas em todas as sociedades industrializadas está se tornando menos ativa fisicamente em suas vidas diárias, passando cada vez mais tempo fazendo atividades sedentárias. $\mathrm{O}$ aumento da atividade física e níveis mais elevados de capacidade de exercício não só reduzirão o risco de desfechos cardiovasculares e diabetes, como também impedirão o desenvolvimento de hipertensão. ${ }^{7}$ A incidência de HA mostrou-se reduzida em $28 \%$ entre homens e $35 \%$ entre mulheres em atividade física, como corrida ou natação, em um seguimento prospectivo de 11 anos, com mais de 12.000 finlandeses. ${ }^{8}$ Em estudo prospectivo longitudinal, ${ }^{9}$ avaliamos a associação entre o nível de atividade física e mortalidade em 200 pacientes hipertensos e diabéticos em 2012 e reavaliados em 2018. Durante 6 anos de seguimento, $80 \%$ dos pacientes ativos sobreviveram em comparação aos sedentários. Além disso, observou-se o benefício da atividade física em pessoas irregularmente ativas, com $65 \%$ de chances de sobreviver em comparação com pacientes que não mantêm essa prática saudável.

O artigo "Medidas Hipertensivas em Escolares: Risco da Obesidade Central e Efeito Protetor da Atividade Física Moderada-Vigorosa", ${ }^{10}$ publicado nesta revista, tem como objetivo verificar a associação de HA, obesidade central e geral e o nível de atividade física em crianças em idade escolar e adolescentes. O estudo envolveu 336 crianças e adolescentes, com idades entre 11 e 17 anos. Foram medidos a altura, peso, circunferência abdominal (CA) e pressão arterial (PA). Calculou-se o escore Z do índice de massa corporal (IMC-Z). O nível de atividade física foi avaliado pela versão curta do Questionário Internacional de Atividade Física (International Physical Activity Questionnaire — IPAQ), de acordo com a prática de atividades físicas moderadas a vigorosas (AF-mv). Crianças com PA sistólica (PAS) ou PA diastólica (PAD) acima do percentil 95, de acordo com sexo, idade e altura ou acima de 120/80 mmHg, foram consideradas hipertensas. A análise estatística foi avaliada pelo teste t de Student, quiquadrado, Mann-Whitney e modelo de regressão logística binária, onde $p<0,05$ foi o nível de significância. Encontrouse $\mathrm{HA}$ em $40,5 \%$ dessa população, sobrepeso em $31,1 \%$ e obesidade em 12,5\%, sendo 13,4\% com maior CA. Crianças insuficientemente ativas em AF-mv representavam 40,2\%. A HA esteve associada a maior CC (OR=6,1; IC 95\%: 2,6 a 14,4) e sobrepeso (OR=2,9; $\mathrm{Cl} 95 \%: 1,8$ a 4,5). Além disso, as crianças com prática de AF-mv apresentaram menor risco de PAD elevada (OR=0,33; IC 95\%: 0,15 a 0,72), redução de risco em 33\%. Em conclusão, obesidade central, obesidade em geral e sexo masculino foram melhores preditores de HA. A 
prática de AF-mv protege as crianças em idade escolar contra o desenvolvimento de hipertensão diastólica.

A obesidade infantil e adolescente é seguida de obesidade na idade adulta e tem estado implicada em diversas doenças crônicas, como diabetes tipo 2, hipertensão e doenças cardiovasculares, e ligada à mortalidade e óbito prematuro. Por esses motivos, nós compartilhamos das mesmas percepções expressas por Tozo et al., ${ }^{10}$ que deixam clara a relevância da obesidade e da hipertensão na infância e na adolescência. Embora a prevalência da hipertensão não seja o objetivo do estudo, é incomum em comparação com o estudo ERICA. ${ }^{5}$ No entanto, o mérito do estudo é

\section{Referências}

1. Chockalingam Impact of world Hypertension day. Can J Cardiol 2007, 23(7): 517-9.

2. Lurbe E, Agabiti-Rosei E, CruickshankJK, DominiczakA, ErdineS, Hirth A, etal. European Society of Hypertension guidelines for the management of high blood pressure in children and adolescents. J Hypertens. 2016;34(10):1887-920.

3. de Almeida DB, Habermann F, Soares VA, Filho RC, Ferreira ES, Junior $\mathrm{AO}$, et al. Comparative study of arterial pressure and prevalence of arterial hypertension in 2 successive cohorts (1975-1976) of students from 16 to 25 years old, Botucatu, SP, Brazil. Rev Saude Publica.1978;12(4):497-505.

4. Bloch KV, Klein CH, Szklo M. ERICA: prevalence of hypertension in Brazilian adolescents. Rev Saúde Publica.2016;50(Suppl 1):9s.

5. Lousada MLC, Martins APB, Canella OS. Ultra-processed foods and the nutritional dietary profile in Brazil. Rev Saúde Publica.2015;49:38.

6. Williams PT. A cohort study of incident hypertension in relation to change in vigorous physical activity in men and women. J Hypertens.2008;26(6):1085-93. a informação preciosa que alerta e nos chama a abrir os olhos para o futuro próximo, enfatizando a importância de fatores crescentes de doenças cardiovasculares como obesidade, hipertensão, estilo de vida sedentário, maus hábitos alimentares e inatividade física que assolam a nossa juventude. Recentemente, aprendemos que os mesmos fatores de risco para doenças cardiovasculares também são os mesmos para doenças causadas por infecção por vírus. ${ }^{11}$ Está na hora de nos mexermos. A mensagem final é: Olhe para o futuro para tomar as decisões corretas no momento, para não se arrepender das consequências causadas pela obesidade e hipertensão na juventude.

7. Leary SD, Ness AR, Smith GD, Mattocks C, Deerek K, Blair SN, et al. Physical activity and blood pressure in childhood: Findings from a population-based study. Hypertension.2008;51(1):92-8.

8. Barengo NC, Hu G, Kastarinen M, Lakka T, Pekkarinen H, Nessinen A, et al. Low physical activity as a predictor of antihypertensive drug treatment in 25-64-years-old population in eastern and south-western Finland. Hypertens.2005(2):293-9.

9. Nunes GFS, Franco RJS, Nicolau NV. Impact of physical activity on survival in hypertensive and diabetic patients in the interior of São Paulo. J Hypertens. 2020;9:1 (open access)

10. Tozo TA, Pereira BO, Menezes Junior FJ, Montenegro CM, Moreira CMM Leite N. Medidas Hipertensivas em Escolares: Risco da Obesidade Central e Efeito Protetor da Atividade Física Moderada-Vigorosa. Arq Bras Cardiol. 2020; 115(1):42-49.

11. Brambilla I, Tosca MA, Fillipo MD. Special issues for COVID-19 in children and adolescentes. Obesity. 2020;May 12: doi:10.1002/oby/22878 (ahead of print) 